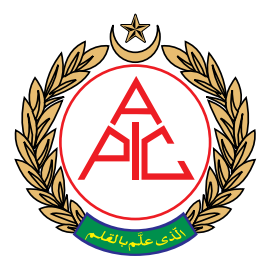

${ }^{1}$ Department of Anesthesiology \& Reanimatology, University of Fukui Hospital, Fukui, (Japan) 2Department of Anesthesiology, Center Hospital of the National Center for Global Health and Medicine, Tokyo, (Japan) ${ }^{3}$ Ventilator \& Anesthesia Device Business Operations, NIHON KOHDEN Corporation, Tokyo, (Japan)

E-mail: ymatsuki@u-fukui.ac.jp o-nagata@fa2.so-net.ne.jp, Yoshihiro_Ogino@mb1.nkc. co.jp, kshigemi@u-fukui.ac.jp Correspondence: Yuka Matsuki MD,

Received: 01 May 2019, Reviewed: 12 September 2019, Accepted: 2 October 2019

\title{
Comparison of propofol administration regulation by pharmacodynamic indicator esTEC (estimated target-effect- site concentration) versus BIS value
}

\author{
Yuka Matsuki ${ }^{1}$, Osamu Nagata ${ }^{2}$, Yoshihiro Ogino ${ }^{3}$, Yuko Nakanishi ${ }^{1}$, \\ Kenji Shigemi ${ }^{1}$
}

\begin{abstract}
Background: Using an algorithm by which the effect-site concentration of propofol (esTEC) necessary for BIS level set from information input from BIS monitor and target controlled infusion (TCI) pump is estimated, we evaluated the accuracy of administration of propofol guided esTEC, whether it is equivalent to that guided BIS value.
\end{abstract}

Methodology: The clinical data of 29 adult patients, who received head/neck surgery and 27 adult patients, who received gynecological surgery, in our hospital from February 2016 and December 2017 were analyzed. Patients were divided into 2 groups: BIS group (adjustment the propofol dose using a target BIS index of 35 to 55, head/ neck surgery $n=15$, gynecological surgery $n=14$ ); and esTEC group (adjustment the propofol based on esTEC ${ }_{45^{\prime}}$, head/neck surgery $n=14$, gynecological surgery $n=13$ ). The relevant data from 2 groups were collected and analyzed.

For statistical analysis, the unpaired t-test, chi-squared test and Wilcoxon test were used, and $p<0.05$ was considered statistically significant. Results were expressed as means \pm standard deviation.

Results: For head and neck surgery, the percentage of time with BIS ${ }_{35-55}$ was significantly greater in the esTEC group than the BIS group $(95 \pm 5 \%$ and $81 \pm 18 \%$, respectively, $\mathrm{p}$ $<0.01)$, as was the percentage of time with BIS ${ }_{<55}(96 \pm 5 \%$ and $86 \pm 17 \%$, respectively, $p=0.0274)$. For gynecological surgery as well, the percentage of time with BIS $_{35-55}$ was significantly higher in the eSTEC than the BIS group $(95 \pm 4 \%$ and $82 \pm 13 \%$, respectively, $\mathrm{p}<0.01)$, as was the percentage of time with BIS ${ }_{<55}(97 \pm 3 \%$ and $88 \pm 15 \%$, respectively, $\mathrm{p}=0.0292)$.

Conclusion: This investigation showed with surgeries at two sites and with patients of different characteristics that propofol dose adjustment based on esTEC could maintain BIS index within the target range similar to adjustment by an attending resident of anesthesia using the BIS index.

Key words: Estimated target effect-site concentration; esTEC; Propofol effect-site concentration; BIS value

Citation: Matsuki Y, Nagata O, Ogino Y, Nakanishi Y, Shigemi K. Comparison of propofol administration regulation by pharmacodynamic indicator esTEC (estimated target-effect-site concentration) versus BIS value. Anaesth pain \& intensive care 2019;23(4):387-391. DOI: 10.35975/apic.v23i4.1178

\section{INTRODUCTION}

In total intravenous anesthesia using propofol, the level of sedation is typically adjusted using the bispectral index (BIS). To accomplish this, the anesthesiologist adjusts the target-controlled 
infusion (TCI) of propofol based on his/her received gynecological surgery in our hospital from

Target BIS value

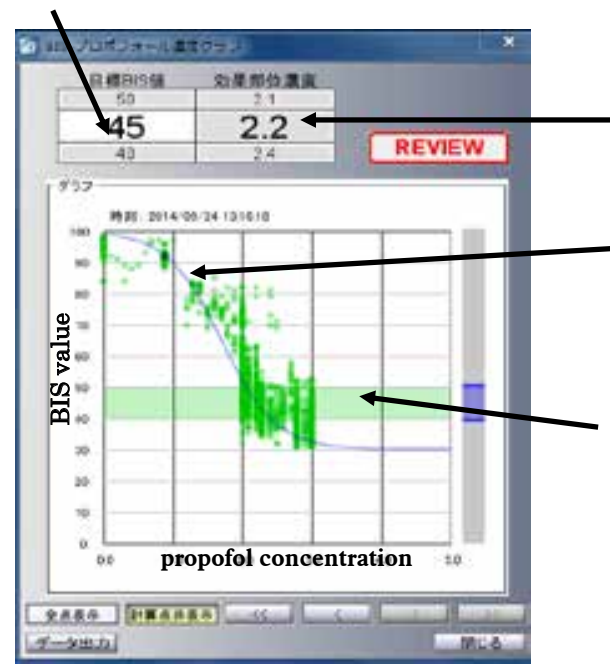

Figure.1 Calculation of esTEC

Using the software installed on the anesthesia management system, the regression curve was estimated in real-time and esTEC was calculated. The esTEC determined at a target BIS level of 45 was labeled esTEC ${ }_{45}$.

experience and intuition. On the other hand, by estimating the real-time regression curve of BIS and propofol effect-site concentration using the software installed in the anesthesia management system, it is possible to determine the estimated target-effectsite concentration (esTEC) of propofol required for achieving the desired effect, and to calculate the amount of propofol required to maintain the designated sedation level objectively and in real-time [1]. However, the pharmacokinetic parameters used to predict the circulating concentration and effectsite concentration are calculated based on population analysis and do not take intersample variations (individual differences) or intrasample variations (surgical invasion) into consideration. For this reason, it is necessary to adjust the propofol target concentration for each individual in order to attain the desired BIS value.

This study compared the percentage of time with $\mathrm{BIS}_{35-55}$ and $\mathrm{BIS}_{<55}$ was maintained intraoperatively between anesthesia management based on esTEC and that based on traditional BIS monitoring in two divergent patient populations undergoing different surgeries.

\section{METHODOLOGY}

This study was approved by the ethics committee of our institution (Approval number: 20160163). The clinical data of 29 adult patients who received head/neck surgery and 27 adult patients who
February 2016 and December 2017 were analyzed. Patients were divided into 2 groups: In the BIS group, the attending residents of anesthesia modified the propofol target concentration setting as a p p r p ri a t e, targeting a BIS value of 35 to 55 . In the esTEC group, the attending residents of anesthesia modified the propofol target concentration setting as appropriate using esTEC $_{45}$ as the index. In both groups, rocuronium was given at appropriate doses according to the attending residents of decision. Patients who simultaneously received volatile anesthesia, regional anesthesia, such as epidural or spinal anesthesia, were pregnant, or were severely obese (body mass index $(\mathrm{BMI})>30 \mathrm{~kg} / \mathrm{m}^{2}$ ) were excluded along with cases in which the collection of data was incomplete. Resident of anesthesia were received anesthesia training with 1 to 3 months.

\section{Anesthetic Protocol:}

Premedication was not given. After entering the operating room, the electrocardiogram, non-invasive sphygmomanometer, pulse oximeter, and BIS Quatro Sensor $^{\circledR}$ for placement on the forehead (Covidien Japan, Tokyo) were connected to the patient, and BIS was measured using a BIS monitor (AE-900P BIS Module, Nihon Kohden, Tokyo, Japan). For anesthesia, total intravenous anesthesia management with propofol, remifentanil and fentanyl was utilized. In both groups, anesthesia was induced with propofol and remifentanil; propofol was infused using the TCI system to a target circulating concentration of $3-4 \mu \mathrm{g} / \mathrm{ml}$ and remifentanil was given at a dose of $0.3-0.5 \mu \mathrm{g} / \mathrm{kg} / \mathrm{min}$. After induction of anesthesia, the continuous infusion rate of remifentanil was adjusted appropriately by the attending resident of anesthesia in both groups based on the patient's hemodynamic parameters and surgical invasiveness.

\section{Definition of esTEC:}


Since the propofol effectsite concentration and resultant sedation effect (BIS index) are known to form an S-curve regression pattern, the target propofol effect-site concentration (ESCp) was calculated in real-time from the logistic function, one of the S-curves. ${ }^{1}$ Using the software installed on Prime GAIA, an automated anesthesia recording system (Nihon Kohden, Tokyo, Japan), paired data of BIS and the calculated effect-site propofol concentration (BIS, ESCp) were collected every 6 seconds, commencing prior to general anesthesia induction. Regression function was determined from the nearest 30 data points in real-time, the propofol effect-site concentration for the corresponding time points was calculated. The propofol effect-site concentration was expressed as esTEC, and the esTEC when the target BIS value of 45 was achieved was identified as "esTEC 45 " (Figure 1).

For each patient, following parameters were retrospectively examined based on the data recorded in the anesthesia management system;
Table 1-Characteristics of patients in the head/neck surgery group

\begin{tabular}{l|c|c|c}
\multicolumn{1}{c|}{ Parameters } & $\begin{array}{c}\text { BIS group } \\
(\mathbf{n = 1 5 )}\end{array}$ & $\begin{array}{c}\text { esTEC group } \\
(\mathbf{n = 1 4 )}\end{array}$ & p value \\
\hline Age (years) & $58 \pm 15$ & $65 \pm 12$ & 0.19 \\
\hline Male sex(\%) & $9(60 \%)$ & $9(64 \%)$ & 0.81 \\
\hline BMl $\left(\mathrm{kg} / \mathrm{m}^{2}\right)^{\mathrm{a}}$ & $22 \pm 5$ & $24 \pm 4$ & 0.36 \\
\hline ASA status (1/11/111) & $3 / 12 / 0$ & $3 / 10 / 1$ & 0.56 \\
\hline Duration of surgery (min) & $132 \pm 43$ & $153 \pm 52$ & 0.26 \\
\hline Duration of anesthesia (min) & $198 \pm 48$ & $221 \pm 52$ & 0.23 \\
\hline Propofol dose $(\mathrm{mg} / \mathrm{kg} / \mathrm{h})$ & $5.3 \pm 1.4$ & $4.0 \pm 0.7$ & $<0.01$ \\
\hline Remifentanil dose $(\mathrm{ug} / \mathrm{kg} / \mathrm{min})$ & $0.18 \pm 0.45$ & $0.20 \pm 0.08$ & 0.42 \\
\hline
\end{tabular}

aMean + SD. BMI; Body mass index ASA; American Society of Anesthesiologists

Table 2. Characteristics of patients in the gynecological surgery group

\begin{tabular}{|c|c|c|c|}
\hline Parameters & $\begin{array}{l}\text { BIS group } \\
(n=14)\end{array}$ & $\begin{array}{l}\text { esTEC group } \\
\qquad(n=13)\end{array}$ & p value \\
\hline Age (years) ${ }^{\mathrm{a}}$ & $47 \pm 16$ & $55 \pm 12$ & 0.18 \\
\hline BMl $\left(\mathrm{kg} / \mathrm{m}^{2}\right)^{\mathrm{a}}$ & $23 \pm 4$ & $22 \pm 3$ & 0.47 \\
\hline ASA status $(1 / 11 / 111)$ & $5 / 7 / 2$ & $2 / 11 / 0$ & 0.13 \\
\hline Duration of surgery (min) & $221 \pm 66$ & $191 \pm 56$ & 0.22 \\
\hline Duration of anesthesia (min) & $295 \pm 68$ & $265 \pm 62$ & 0.234 \\
\hline Propofol does (mg/kg/h) & $5.1 \pm 0.1$ & $4.4 \pm 0.8$ & 0.04 \\
\hline Remifentanil dose (ug/kg/min) & $0.23 \pm 0.05$ & $0.22 \pm 0.49$ & 0.59 \\
\hline
\end{tabular}

settings based on the system display in the esTEC group, we postulated that it would be possible to more accurately adjust the effect-site concentration with mechanically-controlled esTEC and excluded the times at which the effect-site concentration was divergent from the esTEC. Appropriate sedation was defined as a BIS value of 35 to 55 and safe sedation
1. Duration of surgery

2. Duration of BIS $<35$

3. Duration of BIS $>55$

4. Time for which (ESCp - esTEC $\left._{45}\right) /$ esTEC $_{45}$ was $<$ $10 \%$ during $\mathrm{BIS}<35$, and

5. The time for which $\left(\mathrm{ESCp}^{-} \mathrm{esTEC}_{45}\right) / \mathrm{esTEC}_{45}$ was $<10 \%$ during BIS $>55$

To exclude artefacts caused by an electric scalpel or electromyogram, the data and time for which signal quality index (SQI) was $<90$ were excluded from analysis. Moreover, since the anesthesiologist adjusted the TCI pump
Table 3. Percentage of time with BIS $_{35-55}$ were achieved in the head/neck surgery group
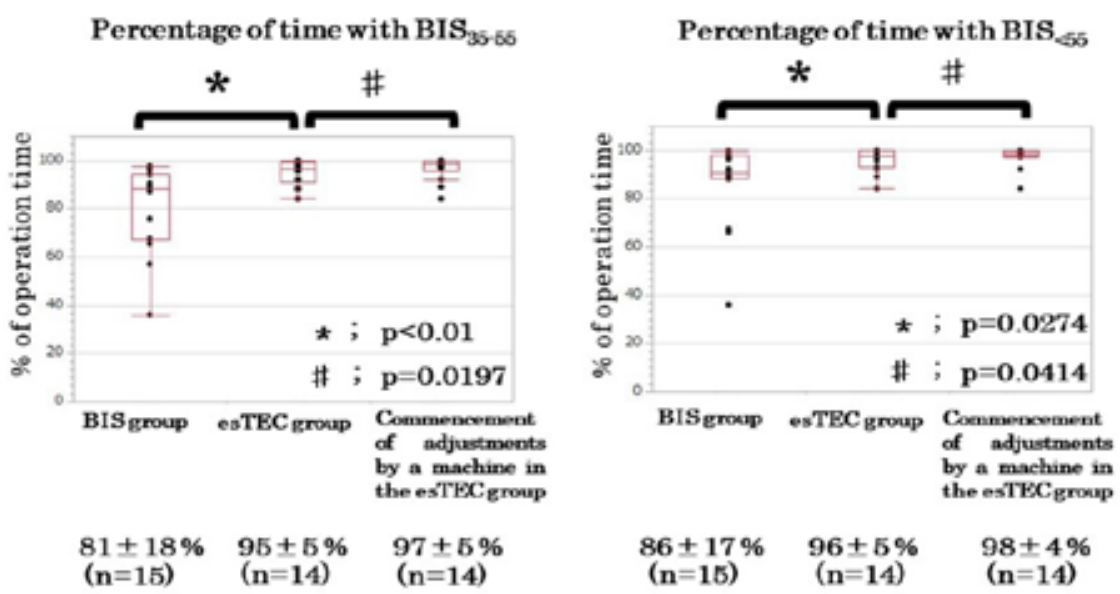
Table 4. Percentage of time with BIS ${ }_{4 \infty}$ were achieved in the gynecological surgery group

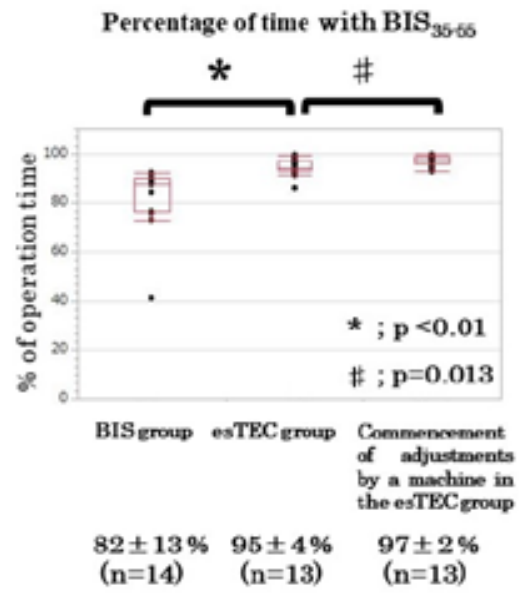

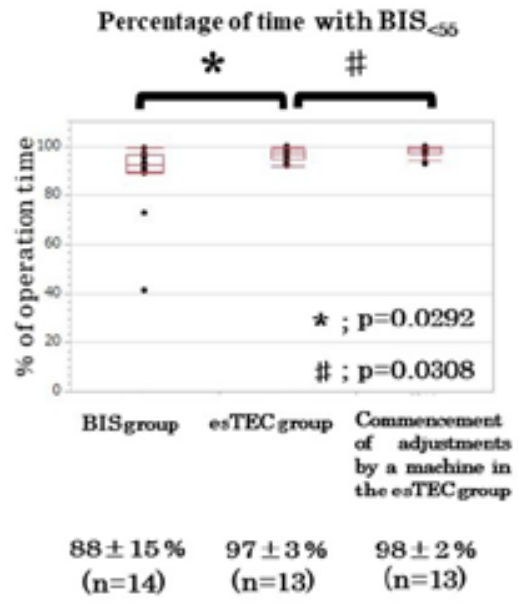

achieved in both groups is shown in Table 3 for head and neck surgery and Table 4 for gynecological surgery. For head and neck surgery, the percentage of time with BIS $18 \%$ and $95 \pm 5 \%$ in the BIS and esTEC groups, respectively, which was significantly greater in the esTEC group $(\mathrm{p}<0.01)$. The percentage of time with BIS $_{35-55}$ reached $97 \pm 5 \%$ when mechanically-controlled esTEC was used, indicating further improvement in the precision of control $(P=0.0197)$. In addition, the percentage of BIS $_{<55}$ was $86 \pm 17 \%$ and $96 \pm$ $5 \%$ for BIS and esTEC groups, respectively, which was again significantly greater in the esTEC group $(\mathrm{p}=0.0274)$. The

was defined as BIS of $\leq 55$. Using parameters (1) to (5), the following values were determined for both groups:

A. Percentage of time with BIS $_{35-55}$ was achieved = [(1)-(2)-(3) $) /(1) \times 100]$,

B. Percentage of time with BIS $_{<55}$ was achieved = [(1)-(3) $) /(1) \times 100]$,

C. Percentage of time of $\mathrm{BIS}_{35-55}$ with the assumption of mechanically-controlled esTEC $==$ [(1)-(4)(5) $) /(1) \times 100]$,

D. Percentage of time of BIS ${ }_{<5}$ with the assumption of mechanically-controlled esTEC $=[(1)-(5)) /(1) \times 100]$

The values of $\mathrm{A}$ to $\mathrm{D}$ were compared between two groups.

For statistical analysis, the unpaired t-test, chisquared test and Wilcoxon test were used, and $\mathrm{P}<0.05$ was considered statistically significant. Results were expressed as means \pm standard deviation.

\section{RESULTS}

Table 1 shows the characteristics of patients who underwent head and neck surgery and Table 2 shows the characteristics of those who underwent gynecological surgery. For both surgeries, there were no differences in age, sex, BMI, American Society of Anesthesiologists grade, surgical procedure time, duration of anesthesia, and remifentanil infusion rate between groups; however, mean propofol infusion rate was significantly lower in the esTEC group for both types of surgeries.

The percentage of time with $\mathrm{BIS}_{35-55}$ and $\mathrm{BIS}_{<55}$ was percentage of time with BIS $<55$ was achieved reached $98 \pm 4 \%$ with the use of mechanically-controlled esTEC, indicating an improvement in precision ( $P$ $=0.0414)$. For gynecological surgery, the percentage of time with BIS ${ }_{35-55}$ was $82 \pm 13 \%$ and $95 \pm 4 \%$ in the BIS and esTEC groups, respectively, which was significantly greater in the esTEC group $(\mathrm{p}<0.01)$. The percentage of time with $\mathrm{BIS}_{35-55}$ increased to

$97 \pm 2 \%$ with the use of mechanically-controlled esTEC, indicating further improvement in the precision $(P=0.013)$. Moreover, the percentage of time with BIS ${ }_{<55}$ was $88 \pm 15 \%$ and $97 \pm 3 \%$ in the BIS and esTEC groups, respectively, which was significantly greater in the esTEC group $(\mathrm{p}=$ 0.0292 ). The percentage of time with BIS ${ }_{<5}$ increased to $98 \pm 2 \%$ with the use of mechanically-controlled esTEC, indicating improvement in the precision ( $P$ $=0.0308$ ).

\section{DISCUSSION}

The results of this study demonstrated that BIS index can be better maintained within the target range by the anesthesiologist adjusting the propofol dose using esTEC rather than BIS as an index, for both head/ neck surgery and gynecological surgery. In addition, this study indicated that more the percentage of time with BIS $_{35-55}$ and BIS ${ }_{<55}$ can be achieved by a machine rapidly and automatically adjusting the propofol dose, rather than residents of anesthesia making adjustments using esTEC as an index. This could be because dose adjustments can be achieved more easily by changing the target concentration using the calculated value of propofol effect-site concentration from the regression function, than by 
having the residents of anesthesia, modify the target concentration by predicting the propofol effect-site concentration based on the BIS index.

Since this trend was observed with both head/neck and gynecological surgery, we postulated that this trend is independent of patient sex or surgery type. Moreover, because the attending anesthesiologist occasionally modified the target concentration while viewing the esTEC display for the esTEC group, it is possible that dose modification may have been neglected because he/she was performing other tasks or was not constantly looking at the monitor. This could be the reason why the BIS value was within the target range for a greater percentage of time with the assumption of mechanically-controlled esTEC.

With both head/neck and gynecological surgery, the esTEC group required a lower propofol dose compared to the BIS group. This was most likely because the BIS index could be properly maintained within the target range in the esTEC group without overdosing or underdosing of propofol.

\section{Possibility of a closed-loop:}

Anesthesia management by a closed-loop system, where there is direct feedback of the measured drug effect to the automatic drug infusion device, allows administration of the drug in accordance with both intersample and intrasample variations from both pharmacokinetic and pharmacodynamic perspectives. It has also been previously reported that BIS can be adjusted within the target range by the auto-control of propofol using BIS as an index compared to the conventional manual control technique. ${ }^{1,2}$ If anesthesia management can be achieved with feedback adjustment of the propofol dose based on esTEC, as used in the present study, it would be possible to more precisely and easily adjust BIS within the target range.

\section{LIMITATIONS}

There was significant variation in the type of cases and surgical invasiveness. Moreover, because the study was based on the premise that BIS is appropriate for esTEC calculation, it is necessary to investigate the effects of age and anesthetic agents in future, to determine whether or not the data are skewed by artefacts such as electric scalpel movement and body movement, and to explore methods to resolve such artefacts. Moreover, drug effect-site concentration between the actual measured value and the calculated value obtained from computer simulation would likely be different. Hence, it might be necessary to investigate the results under different conditions, including with massive bleeding and in obese patients.

\section{CONCLUSION}

In conclusion, BIS index was better maintained within the target range with propofol dose adjustments based on esTEC rather than with dose adjustments made by the residents of anesthesiology using BIS as the index. Our results also suggested that the precision of adjustment might increase further with rapid and frequent adjustments made by a machine rather than with manual adjustments by residents of anesthesia.

Conflict of interest: None declared by the authors.

Acknowledgements: This research was supported by the Japan Agency for Medical Research and Development under Grant No. JP18hk0102052.

\section{Authors' contributions:}

YM - manuscript writing, obtained the written consent from the patients

$\mathrm{ON}$ and $\mathrm{YO}$ : developed the system

$\mathrm{ON}$ and KS: conceived the idea, participated in design and coordination, manuscript drafting

\section{REFERENCES}

1. Liu N, Chazot T, Hamada S, Landais

A, Boichut N, Dussaussoy C, et al. Closed-Loop Co-administration of Propofol and Remifentanil Guided by Bispectral Index: A Randomized Multicenter Study. Anesth Analg
2011;112:546-57. [PubMed] DOl: 10.1213/ANE.0b013e318205680b

2. Orliaguet $G A$, Benabbes Lambert $F$, Chazot T, Glasman P, Fischler M, Liu $N$. Feasibility of closed-loop titration of propofol and remifentanil guided by the bispectral monitor in pediatric and adolescent patient: a prospective randomized study. Anesthesiology

2015;122:759-67. [PubMed] DOI: 10.1097/ALN.0000000000000577 